BMJ Open

Sport \&

Exercise

Medicine

\title{
Exercise training and physiological responses to acute stress: study protocol and methodological considerations of a randomised controlled trial
}

\author{
Elin Arvidson, ${ }^{1,2}$ Anna Sjörs Dahlman, ${ }^{1}$ Mats Börjesson, ${ }^{2,3}$ Lennart Gullstrand, ${ }^{2}$ \\ Ingibjörg $\mathrm{H}$ Jonsdottir ${ }^{1,2}$
}

To cite: Arvidson $\mathrm{E}$, Dahlman AS, Börjesson M, et al. Exercise training and physiological responses to acute stress: study protocol and methodological considerations of a randomised controlled trial. BMJ Open Sport \& Exercise Medicine 2018;4:e000393. doi:10.1136/ bmjsem-2018-000393

Accepted 17 July 2018
Check for updates

\section{(C) Author(s) (or their} employer(s)) 2018. Re-use permitted under CC BY-NC. No commercial re-use. See rights and permissions. Published by BMJ.

${ }^{1}$ The Institute of Stress Medicine, Region Västra Götaland, Gothenburg, Sweden ${ }^{2}$ The Department of Food and Nutrition, and Sport Science, University of Gothenburg, Gothenburg, Sweden ${ }^{3}$ The Department of Neuroscience and Physiology, Sahlgrenska Academy and Sahlgrenska University Hospital/ Östra, Gothenburg, Sweden

\section{Correspondence to}

Elin Arvidson; elin.arvidson@ vgregion.se

\section{ABSTRACT}

Background This paper describes the protocol and methodological prerequisites for a randomised controlled exercise intervention. Selected baseline data from the study are also presented, demonstrating some methodological challenges related to exercise intervention trials. The aim of the trial was to study the effects of exercise training on physiological responses to acute psychosocial stress in untrained individuals.

Methods Individuals with a low level of physical activity were invited to participate in an exercise intervention lasting for 6 months. A total of 119 participants were included and went through a peak oxygen uptake test and a psychosocial stress test at baseline. Adrenocorticotropic hormone (ACTH) and cortisol were measured in connection to the stress test to identify the physiological response.

Results Almost $90 \%$ of the participants reported themselves as untrained, but results from the objectively measured oxygen uptake did not seem to correspond to the reported sedentary lifestyle. The primary outcome measures at baseline varied between individuals. The mean change from pre-test to peak value was $214 \%$ for ACTH and $94 \%$ for cortisol. Of these, 13 individuals did not respond in ACTH and/or and cortisol.

Discussion Supposedly untrained individuals seeking participation in an exercise intervention might not be as untrained as they report, a methodological consideration of importance when evaluating the effects of training. Another important consideration is related to the primary outcome measure, which should be measurable and possible to affect. Absence of reaction at baseline means that changes can only be detected as an increased reaction.

\section{BACKGROUND}

Exercise training has been shown to reduce the risk of several lifestyle-related diseases and to improve mood, well-being and physical performance. ${ }^{1-4}$ The preventive effects of exercise training regarding depression and other mental health conditions are also well documented. ${ }^{56}$ Thus, many symptoms related to psychosocial stress can be prevented with exercise training, and exercise has been

\section{What are the new findings?}

- The target population of an exercise intervention might be difficult to reach particularly regarding the inclusion criteria stating that the participants should be untrained.

- The individual variability regarding the physiological response to acute stress is large. Some individuals actually do not show any response, which will challenge the interpretations of a randomised controlled trial with the aim of studying physiological response to acute stress.

How might it impact on clinical practice?

- When planning a randomised controlled exercise intervention trial, fitness levels should be objectively measured.

- The primary outcome measure should be measurable and possibility of detecting changes over time should be present.

suggested as an important general buffer for stress. $^{78}$

Knowledge of the mechanisms behind the plausible stress-buffering effects of exercise is, however, still limited. The most widely studied possible physiological mechanism is the so-called cross-stressor adaptation hypothesis described by Sothmann et al in $1996 .{ }^{9}$ The acute physiological response to exercise and psychological stress share the similarities of a potent increase in hypothalamic-pituitaryadrenocortical (HPA) responses. ${ }^{10-12}$ Exercise training leads to adaptations with regard to hormonal responses during acute exercise in trained individuals. ${ }^{13}$ The theory of crossstressor adaptation proposes that similar adaptations are also seen in trained individuals during acute psychosocial stress. ${ }^{9}$ The adaptations are suggested to occur partly on brain level, by regulations of neuroendocrine 
functions, and partly on end-organ level, by feedback modifications and adaptations in the organs.

Previous research with regard to the cross-stressor adaptation hypothesis is, however, not consistent. The majority of existing studies in this field are of cross-sectional design. As far as we know, only one randomised controlled trial (RCT) has been conducted by Klaperski et $a l,{ }^{14}$ which is not surprising given the many methodological challenges related to conducting an RCT. For example, the response in the primary outcome measure must be measurable and changeable in all individuals included in the study. Another prerequisite often applied for exercise interventions is an inclusion criterion that assume the individual to be physically inactive at baseline. This might result in challenges connected to the inclusion of the intended population. However, the advantages of an RCT are several compared with a cross-sectional study, and the results from a well-performed RCT is usually more solid than from cross-sectional studies. In the RCT by Klaperski et $a l,{ }^{14}$ the 12 -week exercise training intervention resulted in significantly decreased responses to the laboratory stressor, compared with the control group, confirming the cross-stressor adaptation hypothesis as valid.

The main aim of this paper is to present an RCT protocol and discuss two important methodological issues related to the conduction of an RCT, by presenting selected baseline results from the trial Acute Stress and Exercise Training Intervention (NCT02051127). The study is designed to investigate the effects of exercise training on the neuroendocrine (HPA -axis) responses to acute laboratory psychosocial stress in untrained individuals. The hypothesis is that the intervention will lead to blunted HPA-axis responses to the stress test.

\section{METHODS/DESIGN}

A 6-month exercise training intervention study was performed at the Institute of Stress Medicine (ISM) in Gothenburg, Sweden. The study was a two-armed RCT with equal allocation of participants to an intervention or a control group. All participants went through oxygen uptake tests and psychosocial stress tests at both baseline and follow-up. The inclusion of participants was running from 2013 to 2016. Information about the study was distributed by advertisement in two major newspapers in the district around Gothenburg in western Sweden, and through noticeboards and social media.

\section{Study population and screening}

Participants were initially screened using an emailed computer-based screening questionnaire, which the participants filled out and returned to ISM. Individuals fulfilling the inclusion criteria (see box 1) were booked for a physical screening at the ISM. This included blood tests, 12-lead resting ECG and collection of anthropometric data. Individuals who were not excluded due to any of the exclusion criteria (see box 1) were booked for baseline measurements, including a peak oxygen uptake
Box 1 Inclusion and exclusion criteria for the randomised controlled trial

Inclusion criteria

- Age 20-50years, essentially healthy (does not have diabetes, cardiovascular disease or mental illness).

- No exercise training within the last year and a self-rated level of physical activity equal to one in the Saltin Grimby Physical Activity Level Scale (for details, see Rödjer et a ${ }^{29}$ ).

- Working or studying at least $50 \%$.

\section{Exclusion criteria}

- Glucose level of $\geq 7 \mathrm{mmol} / \mathrm{L}$ and $\mathrm{HbA} 1 \mathrm{c} \geq 48 \mathrm{mmol} / \mathrm{L}$.

- Diverge resting ECG.

- Blood pressure $>140 / 90 \mathrm{~mm} \mathrm{Hg}$.

- Anaemia $(\mathrm{Hb}<120 \mathrm{~g} / \mathrm{L}$ for women, $<130 \mathrm{~g} / \mathrm{L}$ for men).

- $B M l<18.50 \mathrm{r}>35 \mathrm{~kg} / \mathrm{m}^{2}$.

- Medication with beta-blockers, psychopharmacological drugs, glucocorticoids or asthma medicine.

- Unable to exercise at a relatively high intensity.

$\left(\mathrm{VO}_{2}\right.$ peak) test and a psychosocial stress test (see below for description of baseline measures). After enrolment (see figure 1), 119 participants (58 women and 61 men) completed the baseline tests.

\section{Study procedures}

The baseline measurements were conducted in two sessions with 1 week in between, and all tests were performed between 13:00 and 15:00. The first session included a $\mathrm{VO}_{2}$ peak test, cognitive testing and filling out questionnaires covering demographic data. The second test session included the Trier Social Stress Test (TSST) followed by the randomisation procedures, which was done with sealed envelopes and a 50/50\% distribution between the groups. After 6months, follow-up measures were conducted. The procedures were exactly the same as those used when taking baseline measurements.

\section{Peak oxygen uptake test}

The $\mathrm{VO}_{2}$ peak test was performed at the Centre for Health and Performance at the University of Gothenburg. To avoid any effects of food intake on the day of testing, all participants were served a standardised meal (around $500 \mathrm{kcal}$ ) containing controlled amounts of fat, protein and carbohydrates (20,15 and $65 \mathrm{~g}$, respectively) at least 2 hours before the test. After participants warmed up on the bicycle ergometer (Monark 828 E; Monark Exercise AB, Vansbro, Sweden), resistance was gradually increased, starting at $87.5 \mathrm{~W}$ for women and $105 \mathrm{~W}$ for men, increasing by $17.5 \mathrm{~W}$ every minute until exhaustion. Oxygen uptake was measured with the Jaeger Oxycon Pro metabolic chart (Carefusion, Hoechberg, Germany) in a mixing chamber mode, and heart rate was monitored with a pulse sensor (Polar 300 RS; Polar, Finland).

\section{Trier Social Stress Test}

The TSST is a validated and reliable test that has been shown to affect neurohormonal stress responses. ${ }^{15}$ The 


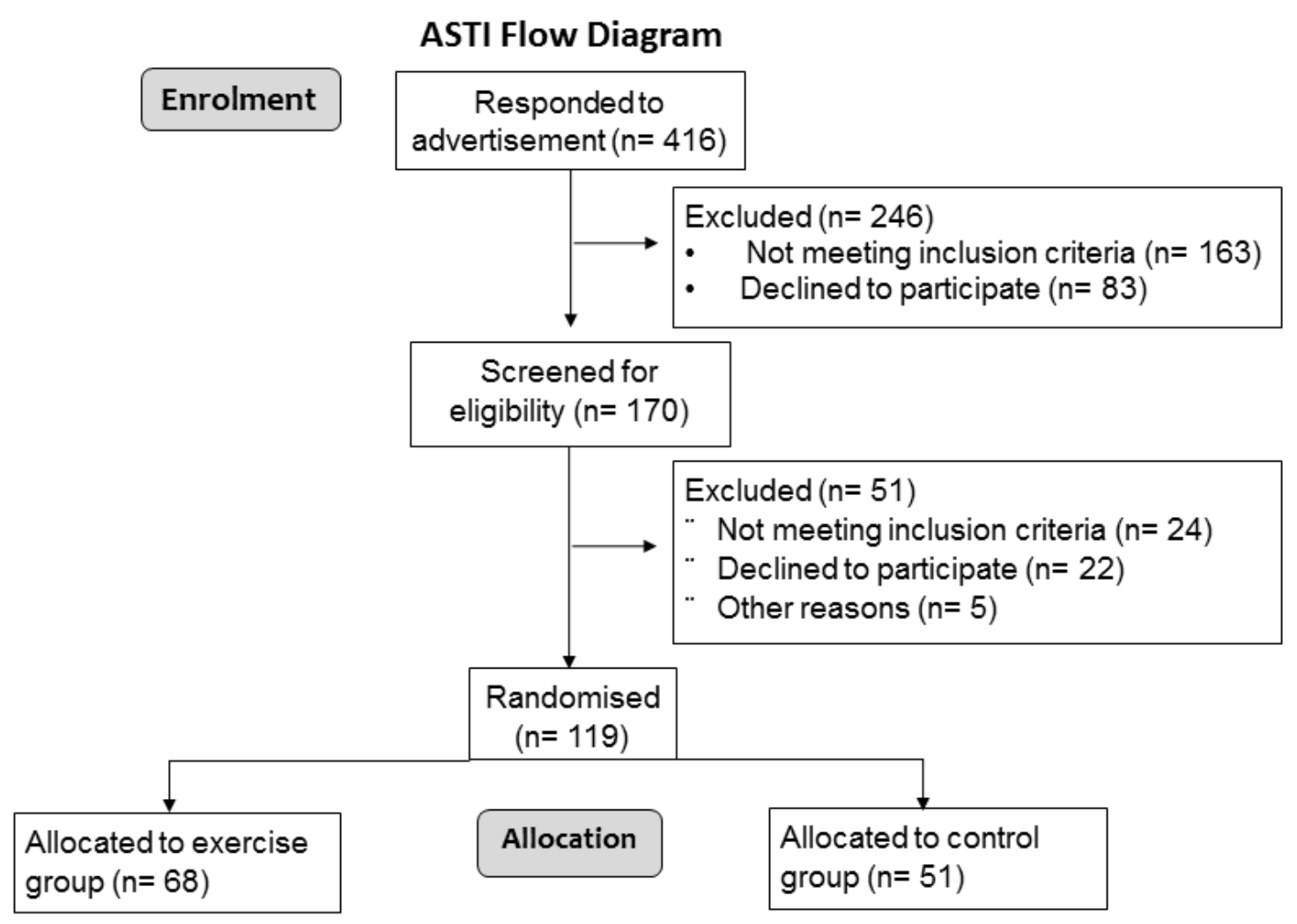

Figure 1 Flow diagram of study enrolment and intervention allocation. ATSI, Acute Stress and exercise Training Intervention.

test is based on two parts: a 5 min free speech and an arithmetic task, both parts being performed in the presence of a three-person committee. The participants are falsely informed that the test is recorded to evaluate their behaviour and performance. Before participants arrived at the institute, they ingested a meal identical to that served before the physical stress test, approximately 2hours before the test. No debriefing was held after the baseline TSST since the test was repeated at the follow-up. However, after the follow-up TSST, the participants received information on the test and a short debriefing.

\section{Exercise intervention}

Participants randomised to the intervention group (IG) will be asked to start out exercise training for 6months. They will be instructed to reach an exercise level of three times per week, 45-60min during each time, reaching an average heart rate of at least $75 \%$ of peak heart rate (measured at the $\mathrm{VO}_{2}$ peak test) $80 \%$ of the work out time. The participants will also be offered lyear of free access to a commercial fitness centre with facilities located in several places in and around the city of Gothenburg. Intensity and duration of the exercise will be monitored using a pulse watch (Garmin 210), and the data will be transferred and registered in an internet-based training $\log$ (www.funbeat.se). The $\log$ will be checked by the coach, and feedback will be offered throughout the training period. The participants will be asked to perform aerobic training of their choice, and to help them achieve the goal of regular exercise, four sessions of Motivational Interviewing will be offered to each participant. The sessions will be led by a trained coach, and the dialogue is guided by Self-determination Theory, ${ }^{16}$ which provides guidelines for the interviewer in the support of participants to increase their level of exercise.

Participants randomised to the control group will be instructed to continue their current level of physical activity between baseline measures and follow-up. After that, they will be encouraged to start exercising. They are offered a motivational group meeting and will receive access to the same fitness centre as the IG after the follow-up. 


\section{Outcome measures and variables}

Primary outcome measures

Neuroendocrine reactions to acute psychosocial stress before and after a 6-month exercise training intervention are the main outcome measures. Adrenocorticotropic hormone (ACTH) and cortisol responses represent HPA-axis reaction.

\section{Measurement of HPA-axis response}

A total of seven samples of plasma ACTH and serum cortisol were collected during each test session. Before the tests, the participants were provided with a peripheral venous catheter in an antecubital vein (BD Venflon Pro, Becton Dickinson Infusion Therapy). To separate plasma, the tubes were cold spun at $3500 \mathrm{rpm}$ for $15 \mathrm{~min}$ and stored at $-80^{\circ} \mathrm{C}$ until analysed. To separate serum, the tubes were spun at $20^{\circ} \mathrm{C}$ for $10 \mathrm{~min}$ at $3500 \mathrm{rpm}$ and stored at $6^{\circ} \mathrm{C}$ until analysis the day after the test. Plasma concentrations of ACTH were measured by immunoradiometric assay (limit of detection, 0.4pmol/L) (CIS bio International, Gif-sur-Yvette Cedex, France). Serum concentrations of cortisol were measured by electrochemiluminescence immunoassay (limit of detection, 0.5nmol/L) (Roche Diagnostics GmbH, Mannheim, Germany).

\section{Secondary outcome measures}

Blood pressure and heart rate before, during and after TSST were measured using an automatic blood pressure cuff (Welch Allyn, ABPM 6100), acquiring data every $5 \mathrm{~min}$. Current level of perceived stress during the TSST was rated with Borg CR 10 Scale of Perceived Stressfulness (13 grades from 'not at all' to 'absolute maximum'). The cognitive test performed was the CNS Vital Signs, ${ }^{17} \mathrm{a}$ computerised test battery comprising seven different tests widely used in previous research studying neurocognitive function. ${ }^{18-20}$ Symptoms of anxiety and depression were evaluated with the Hospital Anxiety and Depression Scale. ${ }^{21}$ The Shirom Melamed Burnout Questionnaire was used to assess levels of burnout, ${ }^{22}$ and the Patient Health Questionnaire assessed the occurrence of somatic symptoms. ${ }^{23} 24$ To evaluate individual strategies for coping with stress, the Coping Inventory for Stressful Situations questionnaire was used, ${ }^{2526}$ and the 14-item questionnaire Perceived Stress Scale was used to evaluate the participants' perceived life stress during the last month. ${ }^{27}$

\section{Statistics}

All randomised participants will be included in initial analyses according to the intention-to-treat procedure. Additional analyses will follow, in accordance with adherence-to-protocol (ATP) procedure where participants with a low compliance will be excluded. The continuous baseline variables age, Body Mass Index, $\mathrm{VO}_{2}$ peak and time-to-exhaustion analyses are presented as means and SD. Group differences at baseline were analysed using independent samples t-tests. ACTH was not normally distributed, but logarithmic transformation enabled the use of a parametric analysis. The categorical variable sex was analysed for group differences using a $\chi^{2}$ test.

The magnitude of response in ACTH and cortisol was calculated by subtracting the pre-test value from the peak value. The per cent change was calculated by dividing the response value with the pre-test value.

A sample size calculation for the main outcome measure cortisol showed that 39 subjects in each group were needed to be able to detect an effect size Cohen's $\mathrm{f}=0.25$, with power $\geq 0.80$ and $\alpha=0.05$ (G*power 3.1 ). Taking into account that a number of drop-outs would occur, the goal was to include 50 subjects in each group.

\section{RESULTS \\ Physiological reactions to acute psychosocial stress}

Basic characteristics of the included participants are shown in table 1. Mean ACTH and cortisol levels at all seven time points are presented in figure 2. The mean response in ACTH from pre-test to peak value was a $214 \%$ increase (range $-42 \%$ to $1860 \%$ ). For cortisol, the mean response was a $94 \%$ increase, ranging from $-20 \%$ to $293 \%$. A decrease was seen in 13 individuals for either ACTH or cortisol, and one individual showed decreased values in both hormones.

\section{Self-reported physical activity level and oxygen uptake}

Self-reported levels of physical activity among the responders to the screening questionnaire and among participants finally included in the study are shown in table 2. The mean baseline $\mathrm{VO}_{2}$ peak values for different age groups among included participants are shown in table 3 .

Table 1 Basic characteristics of the participants at baseline

\begin{tabular}{|c|c|c|c|c|c|c|}
\hline & \multicolumn{3}{|c|}{ Control group } & \multicolumn{3}{|c|}{ Intervention group } \\
\hline & $\mathbf{n}$ & Mean & SD & $\mathbf{n}$ & Mean & SD \\
\hline Age (years) & 52 & 40 & 7.8 & 67 & 38 & 7.2 \\
\hline $\operatorname{sex}(\mathrm{n}+$ ○/ $)$ & 52 & $33 / 34$ & & 67 & $25 / 27$ & \\
\hline BMI (kg/m) & 52 & 25.3 & 4.2 & 67 & 24.8 & 3.3 \\
\hline $\mathrm{VO}_{22}$ peak $\left(\mathrm{mL} \mathrm{kg}^{-1} \mathrm{~min}^{-1}\right)$ & 48 & 32.9 & 6.01 & 61 & 34.2 & 7.1 \\
\hline TTE (min:sec) & 52 & $7: 22$ & $2: 23$ & 67 & $7: 39$ & $2: 19$ \\
\hline
\end{tabular}

BMI, Body Mass Index; TTE, time to exhaustion; $\widehat{\partial}$, men; + , women. 

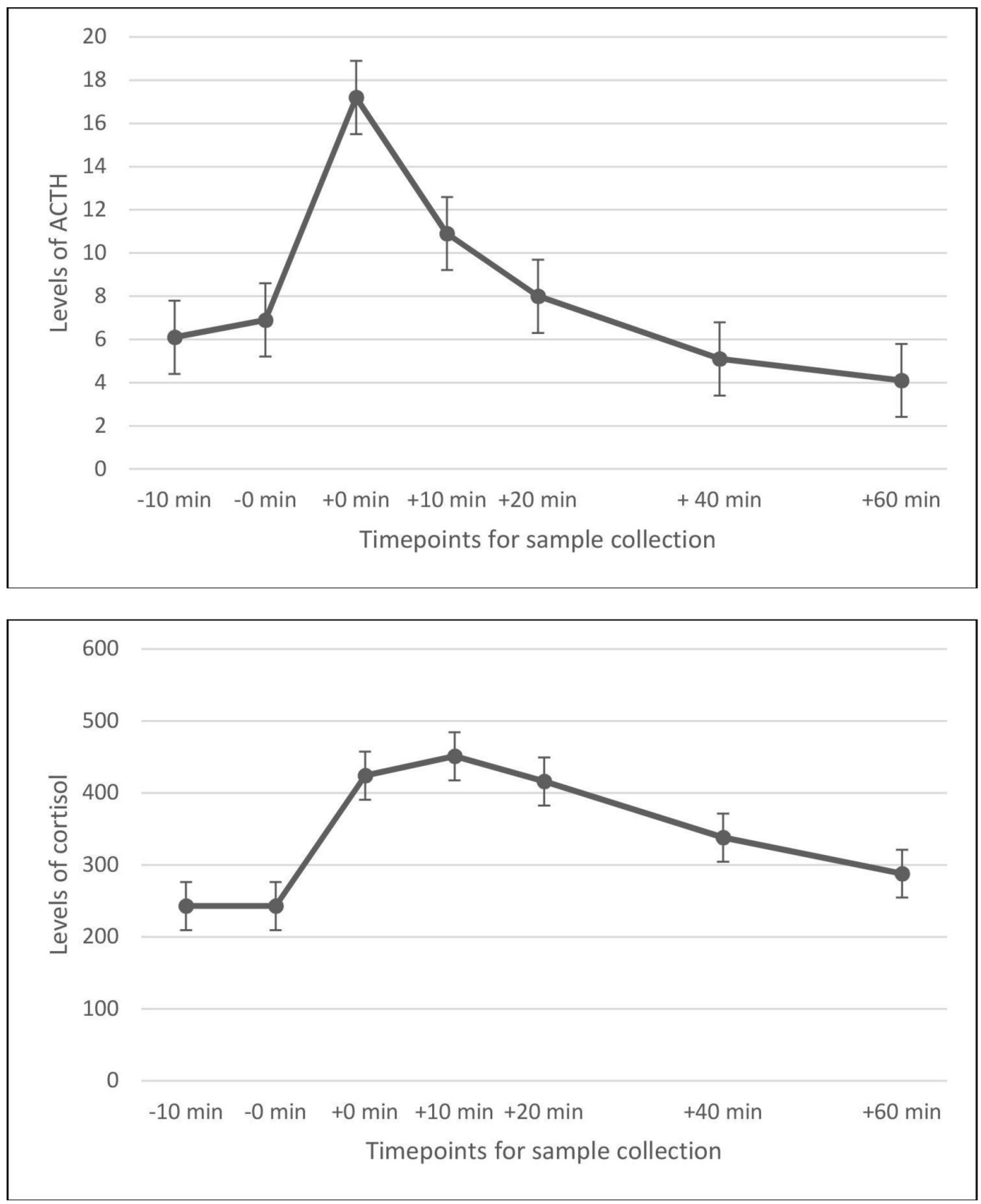

Figure 2 Response of adrenocorticotropic hormone (ACTH) and cortisol to the psychosocial stress test.

\section{DISCUSSION}

\section{Protocol}

Here, we present the protocol of an exercise intervention

Table 2 Reported levels of physical activity at screening and among included participants

\begin{tabular}{|c|c|c|c|c|}
\hline & & $\mathrm{s}$ & LPA & MVPA \\
\hline Responders & $\mathbf{n}$ & n (\%) & n (\%) & n (\%) \\
\hline $\begin{array}{l}\text { Answered } \\
\text { the screening } \\
\text { questionnaire }\end{array}$ & 234 & $189(76.5 \%)$ & $47(20 \%)$ & $8(3.5 \%)$ \\
\hline $\begin{array}{l}\text { Included in } \\
\text { the study }\end{array}$ & 119 & 106(89\%) & $13(11 \%)$ & \\
\hline
\end{tabular}

LPA, light physical activity; MVPA, moderate to vigorous physical activity; S, mostly sedentary. with the aim of investigating the effects of exercise training on neuroendocrine and autonomic responses to acute laboratory psychosocial stress in untrained individuals.

Table 3 Peak oxygen uptake values for different age group

\begin{tabular}{|c|c|c|c|c|}
\hline \multirow[b]{2}{*}{${ }_{2}$ Age group } & \multicolumn{4}{|c|}{ VO22 peak $\left(\mathrm{mL} \mathrm{kg}^{-1} \mathrm{~min}^{-1}\right)$} \\
\hline & $\mathbf{n}$ & i (SD) & $\mathbf{n}$ & $\hat{\partial}$ (SD) \\
\hline $20-29$ & 11 & $36.0(8.7)$ & 5 & $37.3(6.1)$ \\
\hline $30-39$ & 19 & $30.5(8.5)$ & 25 & $38.5(7.5)$ \\
\hline $40-50$ & 28 & $29.4(4.7)$ & 31 & $34.9(5.5)$ \\
\hline
\end{tabular}

ก, men; ㅇ, women. 
The results of the study will add to the knowledge of the mechanisms behind the plausible stress-buffering effects of exercise,which is still limited. The "cross-stressor adaptation hypothesis' will be tested in an RCT design. ${ }^{9}$ The theory of cross-stressor adaptation suggests that adaptation will be seen regarding the response of the HPA axis and the autonomic nervous system during acute psychosocial stress following exercise training. Since previous research is not consistent and most studies in the field are of cross-sectional design, this trial will hopefully add valuable knowledge to the field.

\section{Baseline results}

Conducting an RCT entails a number of methodological challenges. The most prominent is the presence of a valid and stable primary outcome measure. In this paper, we present baseline results of the HPA-axis response to acute laboratory stress, showing that the reaction varies considerably between individuals. An increase in ACTH and cortisol was seen for the majority of the individuals, but the variation was large. In fact, decreased levels were seen in ACTH and/or cortisol in response to acute stress in 13 individuals. The reasons for this result may be several. The most plausible is that the individuals were already affected by stress when they arrived at the laboratory. In these cases, the peak in stress hormones was probably reached before the test started, resulting in a decrease in the levels at the stress test. Another explanation is that the stress stimulus was too weak to cause a physiological response in some subjects, even though the instrument is validated and normally shows a clear reaction in most individuals. ${ }^{28}$ These varying physiological responses to acute stress among the participants constitute methodological challenge. A precondition for all intervention studies is that the primary outcome measure is present and that plausible changes after the intervention are measurable. Since the hypothesis of this study is that a decreased reaction of ACTH and/or cortisol would be seen following the exercise training intervention, this will not be possible to detect in individuals not showing a reaction to the stress test at baseline. As far as we know, this issue is rarely raised, and the large individual variation in the reaction pattern between individuals could partly explain the large variation seen in different previously published studies. This so-called floor effect of an outcome measure is often considered when analysing questionnaires but is less commonly considered in studies of biological reactions. An important methodological consideration is whether these individuals should be removed from ATP analyses.

\section{Who seek participation to the study?}

Further methodological considerations are related to the participants who seek participation in a randomised controlled intervention trial. Several inclusion and exclusion criteria were set, some of which were easy to assess with objective measures whereas other criteria relied on self-reports from the participants. ${ }^{29}$ In the advertisement for the study, we called for individuals who were untrained and had not been regularly physically active during the last year. Nevertheless, $20 \%$ of the respondents to the screening questionnaire reported light physical activity at least 4hours per week, and another $3.5 \%$ reported exercise training at least 2 to 3 hours per week. In the final study population, the individuals reporting regular exercise training were excluded, but due to difficulties in recruiting participants, $11 \%$ of the sample reported a light physical activity level in the Saltin Grimby Physical Activity Level Scale. Still, the majority (89 \%) of the final sample reported themselves as sedentary at screening. This, however, does not seem to be reflected by their oxygen uptake. Compared with reference values for $\mathrm{VO}_{2}$ peak in the general population, ${ }^{30}$ male participants in our study showed an average oxygen uptake, and women showed an oxygen uptake in the upper regions of reference values. Neither men nor women included in the study could thus be defined as 'untrained'. These results indicate that we have not reached the intended target group for the study. Importantly, there is a hereditary component of aerobic fitness $\left(\mathrm{VO}_{o}\right.$ peak) showing that aerobic fitness and physical activity level do not always correspond..$^{31}$ Apparently, we reached individuals reporting a sedentary lifestyle but with an oxygen uptake corresponding to a higher activity level.

\section{Limitations}

Some potential limitations are identified. One of them is related to the stress test. When repeating the test, there is risk for habituation to the test situation. However, previous research has shown that when at least 4months pass between the tests, the risk of habituation is small. ${ }^{32}$ Another potential limitation is related to the exercise intervention. The participants are supposed to exercise on their own, three times per week. Here, the motivation to start out exercise might be an obstacle, but by offering individual motivational dialogues, the initial difficulties hopefully will be facilitated. Also, due to practical reasons, the possibility to blind is restricted, which might be seen as a limitation.

\section{CONCLUSION}

Here, we present an RCT protocol and discuss some important methodological issues regarding conducting an intervention trial. One challenge is to reach the target population, in this study untrained individuals. However, supposedly untrained individuals seeking participation in an exercise intervention might not be as untrained as they report themselves to be. Another important methodological consideration is to ensure that the outcome measures of the study are valid and stable over time. Individual physiological reactions to acute stress vary considerably, and some individuals do not react at all to acute stress. The variability regarding the physiological measures are rarely discussed, and in this case the primary outcome measure of the trial can only be affected in one direction (increase) in several of the participants. We conclude 
that outcome measures of RCTs need to be thoroughly evaluated before the onset of the intervention to ensure a true outcome of the intervention.

Contributors EA: involved in the protocol design and the overall planning of the study. Collected and analysed the data and wrote the manuscript. ASD: involved in the protocol design and the overall planning of the study. Revised the manuscript and provided final approval of the version to be published. MB: involved in the protocol design and the overall planning of the study. Revised the manuscript, added valuable intellectual content and provided final approval of the version to be published. LG: revised the manuscript, added valuable intellectual content and provided final approval of the version to be published. IHJ: involved in the protocol design and the overall planning of the study. Providing substantial contributions to the work and valuable intellectual content, and provided final approval of the version to be published.

Funding This trial was supported by funding from the Swedish Research Council for Health, Working Life and Welfare.

Competing interests None declared.

Patient consent Obtained.

Ethics approval The study was approved by the Regional Ethical Review Board, Gothenburg, Sweden, conducted according to the 1964 Declaration of Helsinki, number 917-12, and registered as a randomised controlled trial at ClinicalTrials. gov (ID no. NCT0251127).

Provenance and peer review Not commissioned; externally peer reviewed.

Open access This is an Open Access article distributed in accordance with the Creative Commons Attribution Non Commercial (CC BY-NC 4.0) license, which permits others to distribute, remix, adapt, build upon this work non-commercially, and license their derivative works on different terms, provided the original work is properly cited and the use is non-commercial. See: http://creativecommons.org/ licenses/by-nc/4.0

\section{REFERENCES}

1. Charlton GA, Crawford MH. Physiologic consequences of training. Cardiol Clin 1997:15:345-54.

2. Szostak J, Laurant P. The forgotten face of regular physical exercise: a 'natural' anti-atherogenic activity. Clin Sci 2011;121:91-106.

3. Duman RS. Neurotrophic factors and regulation of mood: role of exercise, diet and metabolism. Neurobiol Aging 2005;26(Suppl 1):88-93.

4. Rosenwinkel ET, Bloomfield DM, Arwady MA, et al. Exercise and autonomic function in health and cardiovascular disease. Cardiol Clin 2001;19:369-87.

5. Dunn AL, Trivedi MH, Kampert JB, et al. Exercise treatment for depression: efficacy and dose response. Am J Prev Med 2005;28:1-8.

6. Schuch FB, Vancampfort D, Rosenbaum S, et al. Exercise improves physical and psychological quality of life in people with depression: a meta-analysis including the evaluation of control group response. Psychiatry Res 2016;241:47-54.

7. Gerber M, Kellmann M, Hartmann T, et al. Do exercise and fitness buffer against stress among Swiss police and emergency response service officers? Psychol Sport Exerc 2010;11:286-94.

8. Gerber M, Börjesson M, Ljung T, et al. Fitness moderates the relationship between stress and cardiovascular risk factors. Med Sci Sports Exerc 2016;48:2075-81.
9. Sothmann MS, Buckworth J, Claytor RP, et al. Exercise training and the cross-stressor adaptation hypothesis. Exerc Sport Sci Rev 1996;24:267-87.

10. Leal-Cerro A, Gippini A, Amaya MJ, et al. Mechanisms underlying the neuroendocrine response to physical exercise. J Endocrinol Invest 2003;26:879-85.

11. Zschucke E, Renneberg B, Dimeo F, et al. The stress-buffering effect of acute exercise: evidence for HPA axis negative feedback. Psychoneuroendocrinology 2015;51:414-25.

12. Dickerson SS, Kemeny ME. Acute stressors and cortisol responses: a theoretical integration and synthesis of laboratory research. Psychol Bull 2004;130:355-91.

13. Hackney AC. Exercise as a stressor to the human neuroendocrine system. Medicina 2006;42:788-97.

14. Klaperski S, von Dawans B, Heinrichs M, et al. Effects of a 12-week endurance training program on the physiological response to psychosocial stress in men: a randomized controlled trial. J Behav Med 2014;37:1118-33.

15. Kirschbaum C, Pirke KM, Hellhammer DH. The 'Trier Social Stress Test' - a tool for investigating psychobiological stress responses in a laboratory setting. Neuropsychobiology 1993;28:76-81.

16. Fortier MS, Duda JL, Guerin E, et al. Promoting physical activity: development and testing of self-determination theory-based interventions. Int J Behav Nutr Phys Act 2012;9:20.

17. Gualtieri CT, Johnson LG. Reliability and validity of a computerized neurocognitive test battery, CNS Vital Signs. Arch Clin Neuropsychol 2006;21:623-43.

18. Masley S, Roetzheim R, Gualtieri T. Aerobic exercise enhances cognitive flexibility. J Clin Psychol Med Settings 2009;16:186-93.

19. Ingriselli JM, Register-Mihalik JK, Schmidt JD, et al. Outcomes, utility, and feasibility of single task and dual task intervention programs: preliminary implications for post-concussion rehabilitation. J Sci Med Sport 2014;17:580-5.

20. Barker-Collo S, Jones K, Theadom A, et al. Neuropsychological outcome and its correlates in the first year after adult mild traumatic brain injury: a population-based New Zealand study. Brain Inj 2015;29:1604-16.

21. Zigmond AS, Snaith RP. The hospital anxiety and depression scale. Acta Psychiatr Scand 1983;67:361-70.

22. Melamed S, Kushnir T, Shirom A. Burnout and risk factors for cardiovascular diseases. Behav Med 1992;18:53-60.

23. Kroenke K, Spitzer RL, Williams JB. The PHQ-15: validity of a new measure for evaluating the severity of somatic symptoms. Psychosom Med 2002;64:258-66.

24. Kocalevent RD, Hinz A, Brähler E. Standardization of a screening instrument (PHQ-15) for somatization syndromes in the general population. BMC Psychiatry 2013;13:91.

25. Endler NS, Parker JD. Multidimensional assessment of coping: a critical evaluation. J Pers Soc Psychol 1990;58:844-54.

26. Endler NS, Parker JD, Butcher JN. A factor analytic study of coping styles and the MMPI-2 content scales. J Clin Psychol 1993;49:523-7

27. Cohen S, Kamarck T, Mermelstein R. A global measure of perceived stress. J Health Soc Behav 1983;24:385-96.

28. Allen AP, Kennedy PJ, Dockray S, et al. Thetrier social stress test: principles and practice. Neurobiol Stress 2017;6:113-26.

29. Rödjer L, Jonsdottir IH, Rosengren A, et al. Self-reported leisure time physical activity: a useful assessment tool in everyday health care. BMC Public Health 2012;12:693.

30. Koch B, Schäper C, Ittermann T, et al. Reference values for cardiopulmonary exercise testing in healthy volunteers: the SHIP study. Eur Respir J 2009;33:389-97.

31. Williams CJ, Williams MG, Eynon N, et al. Genes to predict $\mathrm{VO}_{2 m}$ trainability: a systematic review. BMC Genomics 2017;18(Suppl ${ }^{2 \max }$ 8):831.

32. Foley P, Kirschbaum C. Human hypothalamus-pituitary-adrenal axis responses to acute psychosocial stress in laboratory settings. Neurosci Biobehav Rev 2010;35:91-6. 